\title{
Lipidomics: A Tool for Studies of Atherosclerosis
}

\author{
Kim Ekroos • Minna Jänis • Kirill Tarasov • \\ Reini Hurme • Reijo Laaksonen
}

Published online: 28 April 2010

(C) The Author(s) 2010. This article is published with open access at Springerlink.com

\begin{abstract}
Lipids, abundant constituents of both the vascular plaque and lipoproteins, play a pivotal role in atherosclerosis. Mass spectrometry-based analysis of lipids, called lipidomics, presents a number of opportunities not only for understanding the cellular processes in health and disease but also in enabling personalized medicine. Lipidomics in its most advanced form is able to quantify hundreds of different molecular lipid species with various structural and functional roles. Unraveling this complexity will improve our understanding of diseases such as atherosclerosis at a level of detail not attainable with classical analytical methods. Improved patient selection, biomarkers for gauging treatment efficacy and safety, and translational models will be facilitated by the lipidomic deliverables. Importantly, lipid-based biomarkers and targets should lead the way as we progress toward more specialized therapeutics.
\end{abstract}

Keywords Lipidomics · Lipid · Molecular lipid ·

Atherosclerosis · Lipoprotein $\cdot$ LDL $\cdot$ HDL $\cdot$ Plaque .

Personalized medicine $\cdot$ Translational medicine .

K. Ekroos $\cdot$ M. Jänis $\cdot$ K. Tarasov $\cdot$ R. Hurme $\cdot$ R. Laaksonen $(\triangle)$

Zora Biosciences Oy,

Biologinkuja 1,

FI-02150 Espoo, Finland

e-mail: reijo.laaksonen@zora.fi

K. Ekroos

e-mail: kim.ekroos@zora.fi

M. Jänis

e-mail: minna.janis@zora.fi

K. Tarasov

e-mail: kirill.tarasov@zora.fi

R. Hurme

e-mail: reini.hurme@zora.fi
Mass spectrometry · Biomarker - Cardiovascular biomarker . Atherosclerosis biomarker $\cdot$ Bioinformatics

\section{Introduction}

Dyslipidemia and resultant atherosclerosis are believed to stem from the imbalance of the lipid metabolites in the affected organism. To this end, advances in mass spectrometry and lipid biochemistry enable the simultaneous highthroughput identification and quantification of hundreds of molecular lipid species in a number of lipid classes that make up the organism's lipidome $[1 \bullet, 2]$. Lipidomic studies quantify the precise chemical constituents of lipidomes, identify lipid cellular distribution, and describe their biochemical mechanisms, interactions and dynamics [3].

Elevated serum total cholesterol and low-density lipoprotein cholesterol (LDL-C) have been established as risk factors for atherosclerosis and its complications. However, a number of coronary artery disease (CAD) or acute myocardial infarction (AMI) patients have LDL-C levels within the recommended range, suggesting the need for additional diagnostic measures of the residual risk. Lipidomics is a tool for differentiating patients based on their molecular lipid profiles. Personalized medicine and diagnostics enabled by lipidomics will facilitate the mission of the right individual receiving the right drug at the right time and dose.

Atherosclerotic plaques are the culmination points of the vascular disease, and retracing the steps of their formation necessitates the structural and functional understanding of the lipoproteins carrying the putative plaque-building blocks as well as the deciphering of the actual plaque composition and function at various stages of its development. Although we may be used to thinking of lipids in the 
context of atherosclerosis as LDL-C, this is a major oversimplification. When traditional clinical chemistry has exhausted its resources, lipidomics provides yet another layer deeper into the patient lipidome, unraveling distinct biochemical characteristics. Indeed, lipidomes of eukaryotic cells comprise hundreds to thousands of molecular lipid species that serve as cellular building blocks (such as cell and organelle membranes), store metabolic energy, and function as bioactive molecules [4-6]. Also, the vascular plaques and lipoproteins responsible for cholesterol transport contain hundreds of other associated lipid species that may exhibit various bioactive properties affecting the course of the disease.

Lipids are physiologic end points of a biological system, and because of their tight regulation they are prime biomarker candidates. Lipidomics in combination with the appropriate clinical samples and biobank material is used today to address the many unmet needs of disease diagnostics. In addition to prognostic and diagnostic value, lipidomics may also find biomarkers that will serve as a read-out of experimental or approved therapies, whereas the appreciation for the bioactivity of lipids has the potential for identifying novel drug targets.

Lipids are also excellent candidates for companion diagnostics in the pharmaceutical arena, which is moving increasingly toward the specialized therapeutics model. Lipidomics has also been used to study translational medicine and thereby is helpful in assessing the utility of various experimental animal models. In this review, we summarize the current state of lipidomics as applied to vascular research and discuss the possibilities it presents to us.

\section{Lipidomics Technology}

\section{Lipidomics and Molecular Lipids}

The recent development of mass spectrometry-driven lipid analysis approaches called "lipidomics" has made it possible to resolve complex lipidomes to their molecular lipid species level at high-throughput and quality required for analyses of clinical cohorts $[1 \bullet, 7,8]$. As a result of the high sensitivity and selectivity of the methods, a lipidomewide analysis of minute sample amounts has become feasible. Present technologies are capable of identifying lipids with different sum compositions (ie, phosphatidylcholine [PC] 34:1) [9], but more important is the identification of molecular lipid species, (eg, PC 16:0/18:1) [10]. In the latter analysis, information of the type of fatty acids and their positions attached to the glycerol backbone making up the particular PC molecule is retrieved. Furthermore, in the same analysis, it is now possible to simultaneously determine the double bond positions of the fatty acids, thus providing information of structurally defined molecular lipid species (eg, PC 16:0/18:1n9) [11•]. Although similar information can be obtained by traditional lipid analysis approaches, such as thin-layer chromatography in combination with gas chromatography, not only does it require much larger sample amounts and more laborious sample preparation, but it does not deliver the molecular lipid species.

The seminal work of Shinzawa-Itoh et al. [12•] showed that the oxygen transfer mechanism in cytochrome $c$ oxidase requires a specific phosphatidylglycerol molecular lipid with palmitate and vaccenate at the $s n-1$ and $s n-2$ positions, respectively, on the glycerol backbone. In line with other studies, this undoubtedly indicates that the lipid structure is an essential determinant of the biological effect. Therefore, molecular lipidomics is an essential asset and the way forward to increase the biological knowledge of molecular lipids and their mechanism of action, supporting both basic research and pharmaceutical drug discovery and development.

Among many lipid classes, the sphingolipids represent a heterogeneous class of biomolecules that can be defined by at least five different long-chain base moieties in mammalian cells, more than 20 species of amidelinked fatty acids, and around 500 different polar head group structures [13, 14]. This level of molecular complexity has been daunting, and therefore much of the biological information still remains unknown. Recently, it has been shown that sphingosine and ceramide promote growth inhibition and apoptosis whereas sphingosine-1phosphate, ceramide-1-phosphate, and other sphingolipids support cell survival, proliferation, differentiation, and migration [15]. Indeed, dysfunctional sphingolipid metabolism has been implicated in numerous diseases including diabetes, atherosclerosis, and hypertension, underscoring the importance of lipid biochemistry for better understanding of the molecular basis of disease [16]. Finally, others have recently unraveled the biological roles of particular molecular sphingolipids, for instance showing that $\mathrm{C} 24$-carbon to $\mathrm{C} 26$-carbon ceramides mediate the death of a $C$. elegans mutant that fails to withstand asphyxia, whereas ceramides with shorter chains have the opposite effect [17].

\section{Shotgun and Targeted Lipidomics}

Proper sample storage, handling, and preparation are crucial in the analytical process for avoiding artificial formation, modification, or loss of lipid species. Therefore, typically lipidomics is performed either by shotgun or targeted approaches. In shotgun lipidomics, lipid extracts are infused directly into a mass spectrometer whereby molecular lipids 
can be identified and quantified using specific precursor ion scans (PIS) and neutral loss scans (NLS) $[18,19]$. The major advantage of this approach is that hundreds of molecular lipids, in nonseparated lipid extracts, can be screened and accurately quantified in less than $30 \mathrm{~min}$. Thus, a simple sample extraction approach like Bligh and Dyer [20], Ekroos [21], and Folch et al. [22] is normally sufficient for a thorough shotgun lipidomic analysis of major lipids such as glycerophospholipids, ceramides, glycerolipids, and sterol lipids (reviewed by Stahlman et al. [2]), which undoubtedly minimizes sample crosscontamination and possible errors originating from laborious and time-consuming sample preparation. Despite the lack of chromatographic preseparation, the selectivity and sensitivity of the analysis are usually not hampered. Today, only a few milligrams of tissue are required for the quantification of hundreds of different molecular lipid species, thus making a lipidome-wide scale analysis of not only whole tissues but also small tissue subsections feasible (Table 1).

There are cases where molecular lipids require preseparation prior to the mass spectrometric analysis. In such circumstances, targeted lipidomics is preferred for enabling the reliable retrieval of high qualitative data. In targeted lipidomics, lipid extracts are separated by liquid chromatography (LC), and eluted lipid species are monitored by online mass spectrometry [23, 24]. Multiple reaction monitoring (MRM) analysis offers both the high selectivity and sensitivity necessary for quantification of low abundant lipid species $[25,26]$. In an optimized setting, the MRM approach can offer the highest detection sensitivity possible to date. However, due to the narrow time window of eluting peaks, especially when using ultra high-performance LC (UHPLC), only a restricted number of MRM transitions are feasible when high qualitative and quantitative data are called for. Thus, targeted lipidomics is best suited for absolute quantification of only a select number of molecular lipids that can be controlled by appropriate lipid standards to minimize possible errors arising from changes in the lipid ionization efficiency.

We believe that both shotgun and targeted lipidomics are the best choices today for deciphering the molecular lipidome comprehensively. Also, the quality and specificity of shotgun and targeted lipidomics are superior and will meet any stringent regulatory quality standards when set-up in the proper environment. In our laboratory, we combine both approaches in parallel to gather the most comprehensive and best quantitative information of the lipidome achievable today. With these technologies, we can currently quantify with high quality and highthroughput up to 2,000 molecular lipids, depending on the particular tissue-specific expression pattern (ie, sample matrix). Simultaneously, these technologies have been recently developed to suit the good laboratory practice and regulatory environment, thus enabling lipidomic analysis of clinical samples.

\section{Biochemist-Friendly Bioinformatics}

Despite the fact that lipidomics is thought of as another "omics" technology, the type of data and information it delivers is considerably different from transcriptomics and proteomics. Measurement of lipid concentrations provides phenotypic information about a state of an organism determined by genetic regulation, functionality of protein machinery, environmental factors, and diet. When a disease or condition is studied, transcriptomics and proteomics will indicate what genes or proteins are affected. Lipidomic measurements, in turn, will bring to light the consequences expressed by lipid concentration changes in biofluid and tissue. Thus, lipidomics provides a readout that is closely connected to the cause of the disease (eg, accumulation of fatty substance in the arteries). It is noteworthy that variations of lipid concentrations within and between individuals are on par with usual clinical chemistry measurements (Table 2).

A challenging task for biochemist/clinician-friendly bioinformatics is to keep the simplicity of interpretation of complex lipid profiles that can be obtained from biofluids and tissues. The first level of analysis will consist of correlating the profiles obtained from controls and diseased or treated patients. Achieving the next level of complexity where patterns of changes in lipid concentrations can be related to specific genes and pathways is more challenging. One approach requires investigation of transcriptional profiles or proteomics experiments conducted together with lipid profiles on the same sample sets. The first attempts for conducting such comparative analysis have been performed by Laaksonen et al. [27] and Gieger et al. [28]. Another approach is to predict affected metabolic functions from a lipid profile itself. For example, involvement of elongases can be predicted by analysis of a ratio of short-chain fatty acids (FA) to long-chain FA. In a similar manner, functionality of saturases/desaturases can be inferred from ratio of lipids that contain FA with a different saturation degree. A reasonable level of abstraction will help to establish rapidly a framework for understanding lipidomic data in a context of acute questions, such as identification of novel noninvasively determined biomarkers and better understanding of complex diseases. With maturation of the bioinformatic approaches in concert with analytical technologies, detailed characterization of lipid pathway-involving kinetic studies will allow us to build more sophisticated models applicable to mammals that are already available for lower organisms [29•]. 
Table 1 Lipidomic platforms and their sample requirements for quantitative molecular species analysis

\begin{tabular}{|c|c|c|c|}
\hline \multirow[t]{2}{*}{ Platform } & \multirow[t]{2}{*}{ Lipid class/molecule ${ }^{\mathrm{a}}$} & \multicolumn{2}{|c|}{ Typical sample amount required } \\
\hline & & Biofluid & Tissue \\
\hline Shotgun & $\begin{array}{l}\text { Cholesteryl ester (CE) } \\
\text { Free cholesterol (FC) } \\
\text { Lysophosphatidic acid (LPA) } \\
\text { Lysophosphatidylcholine (LPC) } \\
\text { Lysophosphatidylethanolamine (LPE) } \\
\text { Lysophosphatidylglycerol (LPG) } \\
\text { Lysophosphatidylinositol (LPI) } \\
\text { Lysophosphatidylserine (LPS) } \\
\text { Phosphatidic acid (PA) } \\
\text { Phosphatidylcholine (PC) } \\
\text { Phosphatidylethanolamine (PE) } \\
\text { Phosphatidylglycerol (PG) } \\
\text { Phosphatidylinositol (PI) } \\
\text { Phosphatidylserine (PS) } \\
\text { Sphingomyelin (SM) } \\
\text { Diacylglycerol (DAG) } \\
\text { Triacylglycerol (TAG) }\end{array}$ & $5 \mu \mathrm{L}$ & $1 \mathrm{mg}$ \\
\hline Targeted ceramides & $\begin{array}{l}\text { Ceramide (Cer) } \\
\text { Glucosylceramide (GlcCer) } \\
\text { Galactosylceramide (GalCer) } \\
\text { Lactosylceramide (LacCer) } \\
\text { Sulfatide (SGalCer) }\end{array}$ & $5 \mu \mathrm{L}$ & $5 \mathrm{mg}$ \\
\hline Targeted gangliosides & $\begin{array}{l}\text { Monosialoganglioside (GM) } \\
\text { Disialoganglioside (GD) } \\
\text { Trisialoganglioside (GT) } \\
\text { Quatrosialoganglioside (GQ) }\end{array}$ & $100 \mu \mathrm{L}$ & $50-100 \mathrm{mg}$ \\
\hline Targeted eicosanoids & $\begin{array}{l}\text { Arachidonic acid (AA) } \\
\text { Leukotriene (LT) } \\
\text { Lipoxine (LX) } \\
\text { Thromboxane (TX) } \\
\text { Prostaglandine (PG) }\end{array}$ & $50-200 \mu \mathrm{L}$ & $20-100 \mathrm{mg}$ \\
\hline Targeted sphingosines & $\begin{array}{l}\text { Sphingosine-1-phosphate (S1P) } \\
\text { Sphinganine-1-phosphate (SA1P) } \\
\text { Sphingosine (SPH) } \\
\text { Sphinganine (SPA) } \\
\text { Phytosphingosine (PSPH) } \\
\text { Ceramide-1-phosphate (Cer1P) }\end{array}$ & $50-100 \mu \mathrm{L}$ & $50-100 \mathrm{mg}$ \\
\hline Targeted fatty acyl coenzyme A & Fatty acyl coenzyme A (FA-CoA) & $50-100 \mu \mathrm{L}$ & $50-100 \mathrm{mg}$ \\
\hline
\end{tabular}

${ }^{a}$ Each lipid class consists of many molecular lipid species. Depending on the matrix, this may be more than 1,000 distinct species

\section{Atherosclerosis: Biology and Lipidomics}

Plaque Biology

Atherosclerosis is a diffuse process of the vascular tree and its development is characterized by the deposition of lipid and blood-borne material to the artery walls. The disease progression is normally slow and silent until the rupture of a vulnerable plaque, causing potentially fatal vascular events. Thus, early identification of individuals at risk is challenging. To that end, sensitive biomarkers with good specificity should be developed. 
Table 2 Select examples of lipidomic platform technical performance

\begin{tabular}{|c|c|c|c|c|}
\hline Platform & Lipid class & $\begin{array}{l}\text { Number of lipid species } \\
\text { detected in human plasma }\end{array}$ & Instrument $\mathrm{CV}, \%^{\mathrm{a}}$ & Process $\mathrm{CV}, \%^{\mathrm{b}}$ \\
\hline \multirow[t]{10}{*}{ Shotgun } & Cholesteryl ester (CE) & 25 & 3 & 21 \\
\hline & Diacylglycerol (DAG) & 24 & 4 & 9 \\
\hline & Lysophosphatidylcholine (LPC) & 10 & 2 & 7 \\
\hline & Phosphatidylcholine (PC) & 50 & 5 & 9 \\
\hline & Ether-linked phosphatidylcholine (PC O) & 66 & 1 & 12 \\
\hline & Phosphatidylethanolamine (PE) & 15 & 8 & 10 \\
\hline & Ether-linked phosphatidylethanolamine (PE O) & 24 & 9 & 15 \\
\hline & Phosphatidylinositol (PI) & 10 & 12 & 14 \\
\hline & Phosphatidylserine (PS) & 3 & 4 & 10 \\
\hline & Sphingomyelin (SM) & 42 & 4 & 9 \\
\hline \multirow[t]{6}{*}{ Ceramides } & Ceramide (Cer) & 8 & 15 & 10 \\
\hline & Glucosylceramide (GlcCer) & 6 & 10 & 7 \\
\hline & Lactosylceramide (LacCer) & 5 & 11 & 23 \\
\hline & Sulfatide (SGalCer) & 25 & 4 & 7 \\
\hline & Total & 313 & & \\
\hline & Average & & 7 & 12 \\
\hline
\end{tabular}

$C V$ coefficient of variation

${ }^{a}$ The instrument and process CV values are presented per lipid class. CV for instrument has been calculated from the same human plasma lipid extract analyzed four times with each platform

${ }^{\mathrm{b}}$ Process CV has been calculated from same mouse brain sample extracted in triplicate

In the Prospective Investigation of the Vasculature in Uppsala Seniors (PIVUS) study, carotid plaques were ultrasound characterized by size and echogenicity, which are both predictors of cardiovascular events [30]. Computerized analysis of carotid plaque size and echogenicity were performed in a population-based manner in 1,016 individuals aged 70 years and compared with traditional risk factors and markers of inflammation. Low highdensity lipoprotein (HDL), increased body mass index (BMI), and decreased glutathione levels were associated with the echolucency of carotid plaques, suggesting the role of metabolic factors in plaque composition. On the other hand, markers of inflammation were related to plaque size alone, implying inflammation to be predominantly associated with the degree of atherosclerosis. The authors suggested that plaque size and echogenicity are influenced by different risk factors.

Fagerberg et al. [31•] studied the heterogeneous structure of carotid atherosclerotic plaques aiming to relate blood flow variations with differences in plaque morphology and composition between sites located upstream and downstream of the maximum stenosis in symptomatic carotid plaques. They observed that the location of maximum stenosis relative to the carotid bifurcation varied considerably between plaques. Furthermore, they reported that as compared with the downstream side, the upstream side of the stenosis had higher incidence of severe lesions with cap rupture and intraplaque hemorrhage, more macrophages, less smooth muscle cells, and more collagen.

The major implication of these findings to plaque studies is that the intraplaque location may be an important confounding factor and that different plaque sections should be studied separately when assessing the lipid building blocks of plaque specimens. We believe that a thorough lipid analysis of atherosclerotic plaques may expose novel markers for atherosclerotic development and plaque rupture prediction. It seems necessary to take the intraplaque location of different lipids into account when analyzing the results of plaque lipidomic screenings [34]. In the best-case scenario, plaque lipids related either to vulnerability or disease development will associate with the corresponding plasma lipid levels, which naturally would increase the clinical utility of such lipid markers. Plaque lipidomics can also be used to study cellular mechanisms and illuminate metabolic pathways related to atherosclerosis, thereby facilitating drug target identification. Current efforts with new drug targets such as phospholipase $\mathrm{A}_{2}\left(\mathrm{PLA}_{2}\right)$ or inflammatory mediators such as eicosanoids are likely to benefit from lipidomic analyses. In our preliminary analyses of human plaque material, we have been able to identify and quantify more than 900 different lipid species, equivalent in number (not composition) to typically lipid-rich tissues such as liver and brain. 
Lipoprotein Trafficking and Metabolism

Lipids are transported to the peripheral tissues and cells and back to the liver by specific carrier particles called lipoproteins. Measuring total levels of serum lipoproteins, LDL, or HDL has been accepted as an indicator of a person's coronary vascular disease (CVD) risk. However, serum fractionation is necessary for investigating the individual properties and possible compositional changes of lipoproteins in different disease states or after a drug therapy. The most widely employed procedures available for isolating serum lipoproteins include different precipitation techniques, sequential flotation ultracentrifugation, size-exclusion gel chromatography, and isopycnic density gradient ultracentrifugation. In recent years, new techniques have arisen for studying the distribution of lipoprotein subfractions that enable, for example, the identification of highly atherogenic small LDL particles [32].

The circulating lipoproteins are heterogenous in structure, shape, and size as well as intravascular metabolism. HDL particles exhibit a number of antiatherogenic properties and play an important role in reverse cholesterol transport (RCT). However, the protective activities of HDL might vary between individuals and under different disease states. For instance, in type 2 diabetes, the endothelial vasoprotective effects of HDL have been shown to be impaired compared with healthy subjects [33]. Moreover, in the study by Ansell et al. [34], HDL isolated from patients with CVD possessed proinflammatory rather than anti-inflammatory properties. Indeed, the lipidomics approach was used to study the properties of small dense $\mathrm{HDL}_{3}$ particles [35•]. The authors demonstrated an elevated sphingosine-1-phosphate to sphingomyelin (S1P/SM) ratio as a feature of $\mathrm{HDL}_{3}$. Moreover, the S1P to SM ratio was shown to correlate positively with antiapoptotic and antioxidative properties of HDL subfractions.

Recently, Hiukka et al. [7] applied current lipidomics technology to investigate the proatherogenic role of apolipoprotein (apo)C-III and demonstrated that apoC-IIIenriched LDL from diabetes patients displayed an altered lipid composition leading to increased susceptibility to the proatherosclerotic sphingomyelinase.

Using immunoprecipitation approach, Bergmark et al. [36] showed that lipoprotein(a) is a preferential carrier of oxidized phospholipids in human plasma. Oxidized lipids are known to play a role in signaling, apoptosis, platelet aggregation, and induction of monocyte adhesion to endothelial cells. Lipidomics can be used to study the physiologic functions and mechanisms of atherogenicity of proatherogenic lipoproteins and to investigate in detail the structural changes induced in lipids under oxidative stress conditions [37].
Animal Models and Translation

To study atherosclerotic plaque and different targeted treatments, a model with good translational properties should be used because the access to human atherosclerotic plaque material is limited, particularly for interventional studies. Lipidomics offer a new tool for disease model selection as it allows detailed comparison of lipid building blocks in plaques obtained from different available animal models. We have compared the lipidomic profiles of the human mammary artery and rat aorta (Laaksonen et al., unpublished data). The data, which are summarized in Fig. 1, indicate that there is a substantial deviation already at the lipid class level between human and rat material. Moreover, detailed analysis of molecular lipid species indicated that the number of recorded molecular lipids and their molecular percentage distribution was quite different between these sample sets. Thus, lipidomic profiling indicated in this case that rat arteries are not a preferential material to study human atherosclerosis due to differently expressed lipids. The rat aorta lipidomics exemplifies the potential of lipidomics for translational medicine.

\section{Diagnostics and Treatment}

\section{Diagnostic Biomarkers of Atherosclerosis}

CVD risk estimation is a difficult task despite improved laboratory and imaging technologies. Traditionally LDL-C and HDL-C measurements have been the cornerstones of lipid-based CVD risk screening. These measures can potentially be improved by direct measurements of apoA-I and/or apoB. Recently, Martin et al. [38] demonstrated that plasma apoB levels, but not LDL-C levels, were associated with coronary artery calcification (CAC) scores in type 2 diabetes patients. Thus, they suggested that apoB levels may be particularly useful in assessing the atherosclerotic burden and cardiovascular risk in type 2 diabetes. However, Ingelson et al. [39] recorded in a large, Framingham population-based cohort that the overall performance of apoB to apoA-I ratio for prediction of CVD was comparable with that of traditional lipid ratios, with no incremental utility over the ratio of total cholesterol to HDL-C.

Furthermore, modified LDL (ie, oxidized LDL [oxLDL]) has been shown to contribute to atherosclerosis development, and that has prompted attempts to use circulating oxLDL as a disease biomarker. Santos et al. [40] examined the relationship of human immunoglobulin $\mathrm{G}(\mathrm{IgG})$ antioxLDL antibodies with cardiovascular disease risk markers in stable individuals and in patients with acute coronary syndrome. They observed that acute inflammatory and metabolic conditions decrease titers of human antibodies of 
Fig. 1 Lipidomic analysis conducted on human left internal mammary artery and rat aorta samples show distinct species-specific composition. $C E$ - cholesteryl esters; $D A G$ - diacylglycerols; $P C$ phosphatidylcholines; $P E$ phosphatidylethanolamines; $P S$ - phosphatidylserines; $S M$ - sphingomyelins

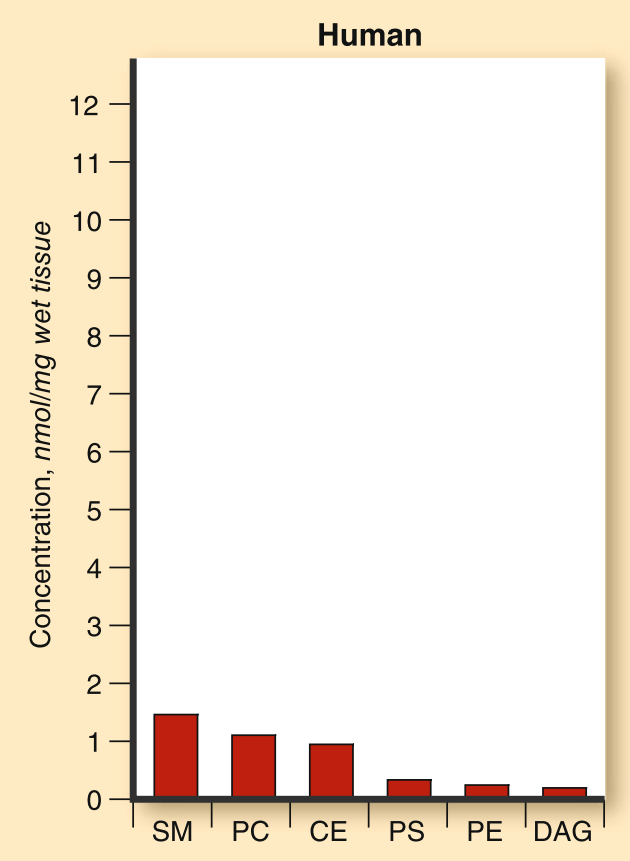

Rat

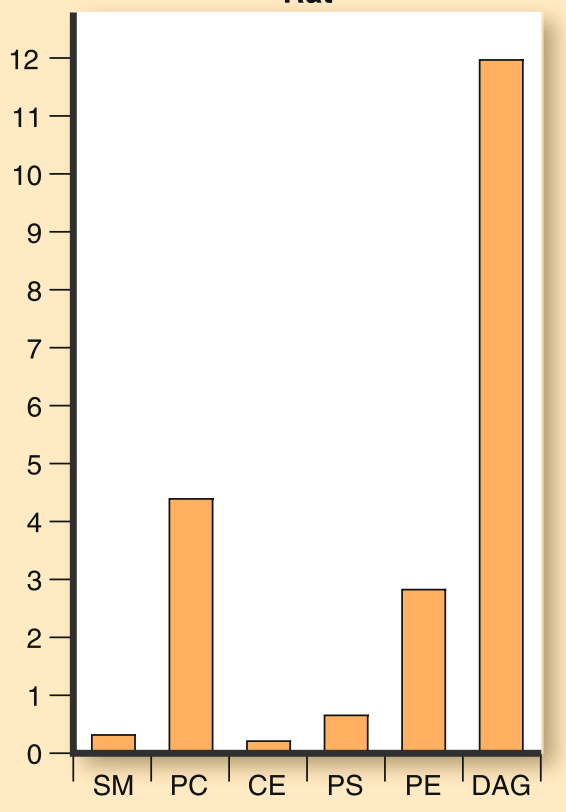

IgG class against oxLDL. They also suggested that circulating anti-oxLDL antibodies could be associated with a protective role in atherosclerosis.

Recently, non-hypothesis-driven metabolomic screenings have been used for the identification of novel CVD biomarkers. Zhang et al. [41] utilized an ultra-fast liquid chromatography coupled with ion trap time-of-flight mass spectrometry (UFLC/MS-IT-TOF) to study plasma and urine metabolic profiles of atherosclerosis rats. Their observations suggest that abnormal metabolism of phenylalanine, tryptophan, bile acids, and amino acids might be related to atherosclerosis development. In addition, Zha et al. [42] identified a metabolic fingerprint of 21 compounds in hamsters that could be a potential marker for the development of atherosclerosis.

Based on our unpublished data, it appears that lipidomics is able to deliver molecular lipids with a greater diagnostic and prognostic value than LDL-C. Detailed lipidomic information will also be useful in tailoring proper treatments to the right patients, and in the best-case scenario new targets may be revealed through pathway mapping enabled by detailed molecular lipid information.

\section{Lipidomics in Drug Trials: Measures of Efficacy and Safety}

To date, lipidomics has not been exploited for the benefit of clinical trials. As new and more precise CVD risk lipid biomarkers are emerging, biochemical monitoring of drug efficacy by traditional LDL-C and HDL-C assays will also be enhanced. Interestingly, lipidomics is able to distinguish the efficacy profiles of various members of a drug class such as statins. In fact, the first attempts to use lipidomic measurements to compare lipid-lowering profiles between different statins have been performed [27, 43]. However, these attempts have so far been more hypothesis generating than informative, as the role of most of the molecular lipids detected in these studies has yet to be established.

Indeed, our vision is that lipidomic analyses will play a significant role in evaluating novel HDL-modulating compounds such as cholesteryl ester transfer protein (CETP) inhibitors. Furthermore, we suspect that current and new LDL-C-lowering treatments and their combinations will be carefully re-evaluated, as it is very likely that these treatments trigger multiple changes in the lipoprotein lipidome despite similar LDL-C-lowering effects.

Lipidomics has also proven to be an efficient way for detecting adverse drug actions. So far, drug-induced liver and muscle toxicities have been demonstrated in plasma lipidomic profiles. The PC to phosphatidylethanolamine (PE) ratio has been observed to reflect hepatocyte membrane fluidity changes, allowing a sensitive measure for liver toxicity [44]. Also, by taking advantage of the molecular lipid information, the authors found new insight into the mechanisms behind the membrane fluidity alterations. We have reported earlier that a distinct plasma lipid pattern can be used to monitor statin-induced gene expression changes in human skeletal muscle and, thus, can detect statin-induced muscle toxicity [27]. Our impression is that this testing can be based on single molecular lipid detection and that these toxicity biomarkers are more sensitive than current markers including liver enzymes and creatine kinase assays. 


\section{Conclusions}

It is apparent that the current lipidomics technology is capable of adding a new dimension of detail and understanding to CVD studies. The CVD pathogenicity is centered on lipid metabolism and the metabolic pathways. The high-precision quantification of molecular lipids is a necessity for deciphering the culprits in the involved cascades. This will be accelerated by the increased application and development of lipidomics technology. Currently, lipidomics attempts to characterize structurally defined molecular lipids from a single analysis [11•] and localize lipids in tissues such as aortic wall [45] and arterial plaques [46]. Maturation of lipidomics and evolution of new lipid-labeling techniques will not only improve lipidome characterization, but also identify the deleterious molecular lipids involved in the dysfunction, where this occurs inside the organism and at what rate. Thus, lipidomics is a prerequisite for advancing the CVD biology and promoting the discovery of cardiovascular disease biomarkers and new drug targets in coming years.

The pharmaceutical industry appears to be shifting gears, moving from the blockbuster approach to more specialist therapies. To be able to cater to a specialist group, care is needed in identifying the correct patients that should receive the medication at the correct time and correct dose. Companion diagnostics, the very rare fruits of personalized medicine, are the sought after tools. In a perfect world, the drug developer would have access to the companion diagnostic biomarker from early stages of research all the way to post-marketing phase of the drug's life cycle. The requirements for these companion diagnostics are not only the ability to identify those patients who will benefit from the therapy (ie, the responders), but to also root out those who are not tolerant of the treatment. Lipidomics offers tools to address both the question of patient selection and stratification as well as the ability to measure the treatment efficacy and safety while taking the drug. To date, we have only a handful of biomarkers employed as true companion diagnostics, which regulatory authorities mandate to be measured prior to drug administration, but none of these are lipid based. The first clinical lipidomics applications will likely be molecular lipid-based CVD risk markers and screening methods that will better separate responders and nonresponders both in clinical trials and clinical practice. As lipidomics is taking an important role in the "omics" hierarchy. We believe that molecular lipids, the tightly regulated metabolic end-products, will provide an important layer of phenotype information that will help us better understand numerous gene-gene, gene-environment, and gene-protein interactions that are involved in the development of atherosclerosis; therefore, lipidomics will be one of the cornerstones in the next generation of mechanistic studies of disease. Finally, it is tempting to speculate on the "theragnostic" potential of combining genomic, transcriptomic, proteomic, and lipidomic data [47]. Although the bioinformatic challenge is substantial, the lipidomic data should be seen as booster for exploiting the strengths of all "omics" in one holistic approach.

Disclosure No potential conflicts of interest relevant to this article were reported.

Open Access This article is distributed under the terms of the Creative Commons Attribution Noncommercial License which permits any noncommercial use, distribution, and reproduction in any medium, provided the original author(s) and source are credited.

\section{References}

Papers of particular interest, published recently, have been highlighted as:

- Of importance

1. • Ejsing CS, Sampaio JL, Surendranath V, et al.: Global analysis of the yeast lipidome by quantitative shotgun mass spectrometry. Proc Natl Acad Sci U S A 2009, 106:2136-2141. This is a comprehensive and pioneering work on the yeast lipidome, showing the road forward for lipidomics.

2. Stahlman M, Ejsing CS, Tarasov K, et al.: High-throughput shotgun lipidomics by quadrupole time-of-flight mass spectrometry. J Chromatogr B Analyt Technol Biomed Life Sci 2009, 877:2664-2772.

3. Han X, Gross RW: Global analyses of cellular lipidomes directly from crude extracts of biological samples by ESI mass spectrometry: a bridge to lipidomics. J Lipid Res 2003, 44:1071-1079.

4. Yetukuri L, Ekroos K, Vidal-Puig A, et al.: Informatics and computational strategies for the study of lipids. Mol Biosyst 2008, 4:121-127.

5. van Meer G: Cellular lipidomics. EMBO J 2005, 24:3159-3165.

6. Wenk MR: The emerging field of lipidomics. Nat Rev Drug Discov 2005, 4:594-610.

7. Hiukka A, Stahlman M, Pettersson C, et al.: ApoCIII-enriched LDL in type 2 diabetes displays altered lipid composition, increased susceptibility for sphingomyelinase, and increased binding to biglycan. Diabetes 2009, 58:2018-2026.

8. Linden D, William-Olsson L, Ahnmark A, et al.: Liver-directed overexpression of mitochondrial glycerol-3-phosphate acyltransferase results in hepatic steatosis, increased triacylglycerol secretion and reduced fatty acid oxidation. FASEB J 2006, 20:434-443.

9. Brugger B, Erben G, Sandhoff R, et al.: Quantitative analysis of biological membrane lipids at the low picomole level by nanoelectrospray ionization tandem mass spectrometry. Proc Natl Acad Sci U S A 1997, 94:2339-2344.

10. Ekroos K, Ejsing CS, Bahr U, et al.: Charting molecular composition of phosphatidylcholines by fatty acid scanning and ion trap MS3 fragmentation. J Lipid Res 2003, 44:2181-2192.

11. - Thomas MC, Mitchell TW, Harman DG, et al.: Ozone-induced dissociation: elucidation of double bond position within massselected lipid ions. Anal Chem 2008, 80:303-311. This article takes lipidomics to a new level of molecular detail, using an 
ozone-based technique for determining double bond position in lipids.

12. - Shinzawa-Itoh K, Aoyama H, Muramoto K, et al.: Structures and physiological roles of 13 integral lipids of bovine heart cytochrome c oxidase. EMBO J 2007, 26:1713-1725. This article shows that molecular lipids and their complexity have specific roles in biology and are not random products. A particular molecular lipid species was shown to be essential to the function of an enzyme.

13. Futerman AH, Hannun YA: The complex life of simple sphingolipids. EMBO Rep 2004, 5:777-782.

14. Zheng W, Kollmeyer J, Symolon H, et al.: Ceramides and other bioactive sphingolipid backbones in health and disease: lipidomic analysis, metabolism and roles in membrane structure, dynamics, signaling and autophagy. Biochim Biophys Acta 2006, 1758: 1864-1884.

15. Hannun YA, Obeid LM: Principles of bioactive lipid signalling: lessons from sphingolipids. Nat Rev Mol Cell Biol 2008, 9:139-150.

16. Alewijnse AE, Peters SL: Sphingolipid signalling in the cardiovascular system: good, bad or both? Eur J Pharmacol 2008, 585:292-302.

17. Menuz V, Howell KS, Gentina S, et al.: Protection of C. elegans from anoxia by HYL-2 ceramide synthase. Science 2009, 324:381-384.

18. Ekroos K, Chernushevich IV, Simons K, et al.: Quantitative profiling of phospholipids by multiple precursor ion scanning on a hybrid quadrupole time-of-flight mass spectrometer. Anal Chem 2002, 74:941-949.

19. Han X, Gross RW: Shotgun lipidomics: electrospray ionization mass spectrometric analysis and quantitation of cellular lipidomes directly from crude extracts of biological samples. Mass Spectrom Rev 2005, 24:367-412.

20. Bligh EG, Dyer WJ: A rapid method of total lipid extraction and purification. Can J Biochem Physiol 1959, 37:911-917.

21. Ekroos K: Unraveling glycerophospholipidomes by lipidomics. In Biomarker Methods in Drug Discovery and Development. Edited by Wang F: New York: Humana Press; 2008:369-384.

22. Folch J, Lees M, Sloane Stanley GH: A simple method for the isolation and purification of total lipides from animal tissues. J Biol Chem 1957, 226:497-509.

23. Kuksis A, Marai L, Myher JJ: Plasma lipid profiling by liquid chromatography with chloride-attachment mass spectrometry. Lipids 1991, 26:240-246.

24. Ogiso H, Suzuki T, Taguchi R: Development of a reverse-phase liquid chromatography electrospray ionization mass spectrometry method for lipidomics, improving detection of phosphatidic acid and phosphatidylserine. Anal Biochem 2008, 375: 124-131.

25. Hopfgartner G, Varesio E, Tschappat V, et al.: Triple quadrupole linear ion trap mass spectrometer for the analysis of small molecules and macromolecules. J Mass Spectrom 2004, 39:845-855.

26. Shaner RL, Allegood JC, Park H, et al.: Quantitative analysis of sphingolipids for lipidomics using triple quadrupole and quadrupole linear ion trap mass spectrometers. J Lipid Res 2009, 50:1692-1707.

27. Laaksonen R, Katajamaa M, Paiva H, et al.: A systems biology strategy reveals biological pathways and plasma biomarker candidates for potentially toxic statin-induced changes in muscle. PLoS ONE 2006, 1:e97.

28. Gieger C, Geistlinger L, Altmaier E, et al.: Genetics meets metabolomics: a genome-wide association study of metabolite profiles in human serum. PLoS Genet 2008, 4:e1000282.

29. - Nielsen J: Systems biology of lipid metabolism: from yeast to human. FEBS Lett 2009, 583:3905-3913. This is an excellent review regarding the similarities of regulation of lipid metabolism between human and yeast and how models can help us understand complex metabolic processes.

30. Andersson J, Sundstrom J, Kurland L, et al.: The carotid artery plaque size and echogenicity are related to different cardiovascular risk factors in the elderly: the Prospective Investigation of the Vasculature in Uppsala Seniors (PIVUS) study. Lipids 2009, 44:397-403.

31. - Fagerberg B, Ryndel M, Kjelldahl J, et al.: Differences in lesion severity and cellular composition between in vivo assessed upstream and downstream sides of human symptomatic carotid atherosclerotic plaques. J Vasc Res 2009, 47:221-230. This article highlights the importance of systemic analysis of plaque samples. The heterogeneous structure of carotid plaques should be taken into account when studying plaque morphology and composition.

32. Moon JY, Kwon HM, Kwon SW, et al.: Lipoprotein(a) and LDL particle size are related to the severity of coronary artery disease. Cardiology 2007, 108:282-289.

33. Sorrentino SA, Besler C, Rohrer L, et al.: Endothelialvasoprotective effects of high-density lipoprotein are impaired in patients with type 2 diabetes mellitus but are improved after extended-release niacin therapy. Circulation 2007, 121:110-122.

34. Ansell BJ, Navab M, Hama S, et al.: Inflammatory/antiinflammatory properties of high-density lipoprotein distinguish patients from control subjects better than high-density lipoprotein cholesterol levels and are favorably affected by simvastatin treatment. Circulation 2003, 108:2751-2756.

35. - Kontush A, Therond P, Zerrad A, et al.: Preferential sphingosine1-phosphate enrichment and sphingomyelin depletion are key features of small dense HDL3 particles: relevance to antiapoptotic and antioxidative activities. Arterioscler Thromb Vasc Biol 2007, 27:1843-1849. The elegant work in this article shows that the structural and functional properties of HDL subfractions will be a key to understanding lipoprotein metabolism.

36. Bergmark C, Dewan A, Orsoni A, et al.: A novel function of lipoprotein [a] as a preferential carrier of oxidized phospholipids in human plasma. J Lipid Res 2008, 49:2230-2239.

37. Domingues MR, Reis A, Domingues P: Mass spectrometry analysis of oxidized phospholipids. Chem Phys Lipids 2008, 156:1-12.

38. Martin SS, Qasim AN, Mehta NN, et al.: Apolipoprotein B but not LDL cholesterol is associated with coronary artery calcification in type 2 diabetic whites. Diabetes 2009, 58:1887-1892.

39. Ingelsson E, Schaefer EJ, Contois JH, et al.: Clinical utility of different lipid measures for prediction of coronary heart disease in men and women. JAMA 2007, 298:776-785.

40. Santos AO, Fonseca FA, Fischer SM, et al.: High circulating autoantibodies against human oxidized low-density lipoprotein are related to stable and lower titers to unstable clinical situation. Clin Chim Acta 2009, 406:113-118.

41. Zhang F, Jia Z, Gao P, et al.: Metabonomics study of atherosclerosis rats by ultra fast liquid chromatography coupled with ion trap-time of flight mass spectrometry. Talanta 2009, 79:836-844.

42. Zha W, Ai J, Wang G, et al.: Metabonomic characterization of early atherosclerosis in hamsters with induced cholesterol. Biomarkers 2009, 14:372-380.

43. Bergheanu SC, Reijmers T, Zwinderman AH, et al.: Lipidomic approach to evaluate rosuvastatin and atorvastatin at various dosages: investigating differential effects among statins. Curr Med Res Opin 2008, 24:2477-2487.

44. Sergent O, Ekroos K, Lefeuvre-Orfila L, et al.: Ximelagatran increases membrane fluidity and changes membrane lipid composition in primary human hepatocytes. Toxicol In Vitro 2009, 23:1305-1310.

45. Malmberg P, Borner K, Chen Y, et al.: Localization of lipids in the aortic wall with imaging TOF-SIMS. Biochim Biophys Acta 2007, 1771:185-195.

46. Manicke NE, Nefliu M, Wu C, et al.: Imaging of lipids in atheroma by desorption electrospray ionization mass spectrometry. Anal Chem 2009, 81:8702-8707.

47. Ozdemir V, Williams-Jones B, Glatt SJ, et al.: Shifting emphasis from pharmacogenomics to theragnostics. Nat Biotechnol 2006, 24:942-946. 